Open Access

Original Article

\title{
Metabolic syndrome in systemic lupus erythematosus was closely related to body mass index, blood pressure, blood sugar, blood lipids, and arthritis
}

\author{
Lai-Run Jin', Meng-Jun Tao ${ }^{2}$, Jun Zhou ${ }^{3}$, Liang $\mathrm{Xu}^{4}$, \\ Qiang $\mathrm{Li}^{5}$, Zhi Li ${ }^{6}$, Hui Peng ${ }^{7}$, Hui Yuan ${ }^{8}$
}

\begin{abstract}
Background and Objective: Prevention and control of metabolic syndrome is the key to improving the development of systemic lupus erythematosus. The aim of this study was to analyze the relevant factors regarding metabolic syndrome (MS) in systemic lupus erythematosus (SLE).

Methods: A total number of 1238 SLE patients in Yijishan Hospital of Wannan Medical College, Anhui province, from February 2012 to July 2017, were analyzed retrospectively. SLE patients with MS were grouped to group SLE-MS, the others without MS was grouped to group SLE-nMS. The two groups were compared with respect to general characteristics, clinical signs, and laboratory parameters. Random forest approach and multivariate logistic regression were conducted to analyze the related factors regarding MS in SLE.

Results: The constituent ratio of metabolic syndrome was $27.14 \%(336 / 1238)$. More SLE patients with MS presented with more farmers, more married people, lower education level, and more lupus nephritis, proteinuria, oral ulcers, tubular urine, hematuria than SLE patients without MS $(P<0.05)$. Moreover, eighteen important variables, whose average importance scores were highest and whose error rates were lowest, were selected by random forest method. Data from multivariate logistic regression showed that MS in SLE was related with BMI, diastolic blood pressure, systolic blood pressure, fasting blood glucose, arthritis, urea, triglycerides, high-density lipoprotein, and white blood cells.

Conclusion: MS in SLE was closely related to BMI, blood pressure, blood sugar, blood lipids, arthritis, white blood cells, and urea. Targeted prevention and conclusion measures for the risk factors should be taken as early as possible.
\end{abstract}

KEYWORDS: Metabolic syndrome, Random forest algorithm, Relevant factors, Systemic lupus erythematosus.

doi: https://doi.org/10.12669/pjms.36.6.2093

How to cite this:

Jin LR, Tao MJ, Zhou J, Xu L, Li Q, Li Z, et al. Metabolic syndrome in systemic lupus erythematosus was closely related to body mass index, blood pressure, blood sugar, blood lipids, and arthritis. Pak J Med Sci. 2020;36(6):1220-1227. doi: https://doi.org/10.12669/pjms.36.6.2093

This is an Open Access article distributed under the terms of the Creative Commons Attribution License (http://creativecommons.org/licenses/by/3.0), which permits unrestricted use, distribution, and reproduction in any medium, provided the original work is properly cited.

Correspondence:

Hui Yuan,

Department of Epidemiology and Biostatistics,

School of Public Health,

Wannan Medical College,

No 22 Road Wenchangxi, Yijiang District,

Wuhu, Anhui, 241002,

PR China.

Email: yuanhui0553@126.com

* Received for Publication:

* $1^{\text {st }}$ Revision Received:

* $2^{\text {nd }}$ Revision Received:

* Final Revision Accepted:

Pak J Med Sci
November 12, 2019

December 13, 2019

July 17,2020

July 20,2020

\section{INTRODUCTION}

Systemic lupus erythematosus (SLE) is an autoimmune-mediated diffuse connective tissue disease characterized by immune inflammation. The incidence of SLE in the world is about (7.4 159.4)/100,000, which seriously endangers the physical and mental health of residents and becomes an important global public health problem. ${ }^{1}$ The majority of SLE patients were women, and the incidence of myocardial infarction, congestive heart failure, and stroke in SLE women 
increased by 8.5 times, 13.2 times, and 10.1 times, respectively, and SLE patients developed menopause 3-4 years earlier than ordinary women. ${ }^{2}$ The incidence of hypertension, diabetes, elevated very low-density lipoprotein, elevated triglycerides and atherosclerosis in SLE patients is significantly higher than that in the general population, and the absolute risk of coronary heart disease in SLE exceeds that in Type-2 diabetes.-5 These studies have shown that the risk of cardiovascular disease (CVD) in SLE patients is significantly higher than in the general population.

The late cause of death in patients with SLE is primarily CVD. The increase in CVD risk in SLE is caused by multiple factors. Selzer et al. selected 214 women with SLE who had no clinical manifestations of cardiovascular disease and found that carotid plaque formation and hypertension were associated with a decrease in high-density lipoprotein. ${ }^{6}$ Hyperlipidemia and hyperglycemia are independent risk factors for intimal thickening, and hardening of the aorta is also associated with hypertension. Studies have found that serum total cholesterol (TC) greater than $5.2 \mathrm{mmol} /$ Lcan predict the occurrence of coronary heart disease (CHD) in SLE patients, $24 \%$ of patients with elevated TC levels have $\mathrm{CHD}$, and only $3 \%$ of patients with normal TC levels developed CHD. ${ }^{7}$ The role of these traditional risk factors in the development of atherosclerosis in SLE patients cannot be ignored, and metabolic syndrome (MS) is just a collection of risk factors, that is, central obesity and insulin resistance as the central link, with hypertension, a group of metabolic disorders with hypertriglyceridemia, impaired glucose tolerance, or type 2 diabetes as the main clinical manifestations. ${ }^{8}$ MS is an independent risk factor for CVD in patients with SLE. So prevention and control of MS is the key to improving the development of SLE., ${ }^{910}$ This study analyzed the data of 1238 patients with SLE in order to explore the relevant factors of MS in SLE patients.

\section{METHODS}

A total of 1238 SLE patients including 143 males and 1095 females, 336 with MS, 902 without MS, with an average age of $41.64 \pm 22.92$ years old, were recruited from Affiliated Yijishan Hospital of Wannan Medical College. All patients were newonset cases first diagnosed by hospital physicians. SLE was defined based on the classification criteria revised by the American College of Rheumatology (ACR) in 1997..1 Disease activity was assessed based on the Systemic Lupus Erythematosus
Disease Activity Index 2000 (SLEDAI)..$^{12}$ MS was defined based on the diagnostic criteria revised by the Chinese diabetes society (CDS) in $2004 .^{13}$ Three or more of the following four components are defined as MS:

a. Overweight or obese, $\mathrm{BMI}^{3} 25 \mathrm{~kg} / \mathrm{m} 2$.

b. Hyperglycemia, fasting blood glucose $>6.1$ mmol / L, or two hour postprandial blood glucose $>17.8 \mathrm{mmol} / \mathrm{L}$, and / or have been diagnosed with diabetes and treated.

c. Hypertension, systolic blood pressure ${ }^{3} 140$ $\mathrm{mmHg}$ and / or diastolic blood pressure ${ }^{3}$ $90 \mathrm{mmHg}$, or have been diagnosed with hypertension and treated.

d. Hypertriglyceridemia, triglyceride ${ }^{3} 1.7 \mathrm{mmol}$ / L, and high-density lipoprotein cholesterol $<0.9 \mathrm{mmol} / \mathrm{L}$ for male, and $<1.0 \mathrm{mmol} / \mathrm{L}$ for female.

Exclusion criteria for all patients were as follows:

1. Patients with anemia.

2. Patients with malignancies.

3. Patients with pregnancy.

4. Patients with viral infectious diseases.

5. Patients with other autoimmune diseases.

General characteristics, clinical signs, and laboratory parameters were obtained through epidemiological interviews and hospital records. Patients were randomly selected from ligible cases in the ward of the hospital's Rheumatology Department based on their hospitalization number and a random number table.

Statistical analysis: Epidata 3.0 software was used to enter data, and SPSS 17.0 software was used for single factor analysis and multivariate analysis. The $\mathrm{R}$ 3.5.1 software RandomForest command package is used for dimensionality reduction and initial screening of variables. We use the random forest algorithm to sort the importance of variables, run the sliding window sequential forward feature selection (SWSFS) process, ${ }^{14}$ plot the error rate graph, and combine the variables with the lowest error rate into the binary logistic regression model. Numerical data conforming to normal distributions were presented as means \pm standard deviation (SD); those not normally distributed were presented as medians (interquartile range, IQR). MannWhitney rank sum tests or Two-tailed Student's $t$-tests for independent samples were performed to estimate differences between groups for continuous variables. The Chi-squared test and Fisher's exact test were used to assess differences in categorical variables among groups. A two-tailed $p$ value $<0.05$ was taken to be statistically significant. 
Table-I Comparison of general information between SLE-MS group and SLE-nMS group [n(\%)].

\begin{tabular}{|c|c|c|c|c|c|}
\hline Variables & $n$ & SLE-MS & SLE-nMS & $\chi^{2}$ & $P$ \\
\hline \multicolumn{6}{|l|}{ Gender } \\
\hline Male & 143 & $41(12.20)$ & $102(10.28)$ & 0.192 & 0.662 \\
\hline Female & 1095 & $295(87.80)$ & $800(80.65)$ & & \\
\hline \multicolumn{6}{|l|}{ Age } \\
\hline $11 \sim$ & 29 & $5(1.49)$ & $24(2.42)$ & 5.391 & 0.145 \\
\hline $18 \sim$ & 560 & $166(49.40)$ & 394 (39.72) & & \\
\hline $45 \sim$ & 319 & $88(26.19)$ & $231(23.29)$ & & \\
\hline 60 & 103 & $21(6.25)$ & $82(8.27)$ & & \\
\hline \multicolumn{6}{|l|}{ Occupation } \\
\hline${ }^{\mathrm{a}}$ Farmer & 272 & $85(25.30)$ & $187(18.85)$ & 16.185 & 0.003 \\
\hline ac Unemployed & 552 & $167(49.70)$ & $385(38.81)$ & & \\
\hline bc Worker & 125 & $26(7.74)$ & $99(9.98)$ & & \\
\hline bc Student & 57 & $8(2.38)$ & $49(4.94)$ & & \\
\hline${ }^{\mathrm{b}}$ Other & 232 & $50(14.88)$ & $182(18.35)$ & & \\
\hline \multicolumn{6}{|l|}{ Marital status } \\
\hline a Married & 1062 & 305 (90.77) & 757 (83.92) & 11.766 & 0.003 \\
\hline bc Unmarried & 169 & $28(8.33)$ & 141 (15.63) & & \\
\hline ac Divorced or Widowed & 7 & $3(0.89)$ & $4(0.44)$ & & \\
\hline \multicolumn{6}{|l|}{ Educational level } \\
\hline a Elementary school and below & 382 & $165(49.11)$ & $217(24.06)$ & 72.793 & $<0.001$ \\
\hline b Middle school & 625 & 130 (38.69) & $495(54.88)$ & & \\
\hline${ }^{\mathrm{b}}$ College and above & 231 & $41(12.20)$ & 190 (19.15) & & \\
\hline
\end{tabular}

Notes: Different superscript letters denote significant differences $(\mathrm{P}<0.05)$.

Significant results are highlighted in bold type.

Ethics approval and consent to participate: The study was approved by the Ethics Board of Wannan Medical College (Number: 2016032; date: December 15, 2015). All patients gave informed consent for publication in the research study and patient privacy was protected.

\section{RESULTS}

Participants with MS accounted for $27.14 \%$ of SLE patients. The age $(P=0.145)$ and gender $(P=0.662)$ between the SLE-MS group and the SLE-nMS group were not statistically different. The proportion of farmers, married people and low education level in the SLE-MS group was statistically higher than that in the SLE-nMS group $(P<0.05)$ (Table-I).

The proportion of participants with lupus nephritis (LN), proteinuria, mouth ulcers, Cylindruria and hematuria in the SLE-MS group was statistically higher than that in the SLE-nMS group (all $P<0.05$ ) (Table-II).

Body mass index (BMI), SLE disease activity index (SLEDAI), systolic blood pressure (SBP), diastolic blood pressure (DBP), white blood cell count (WBC), mean red blood cell volume (MCV), red blood cell distribution width (RDW), urine microalbumin, urine red blood cell count (URBC), urinary tube type count (UTT), total protein (TP), triglyceride (TG), albumin (ALB), high density lipoprotein (HDL), globulin (GLB), low density lipoprotein (LDL), aspartate aminotransferase (AST), complement $3(\mathrm{C} 3)$, urea (UA), creatinine (CREA), immunoglobulin A (IgA), fasting blood glucose (GLU), immunoglobulin $\mathrm{G}(\mathrm{IgG})$, total cholesterol (TC) and 24h urine protein (UP24) in SLE-MS group were statistically different from that in SLE-nMS group (all $P<0.05$ ) (Table-III).

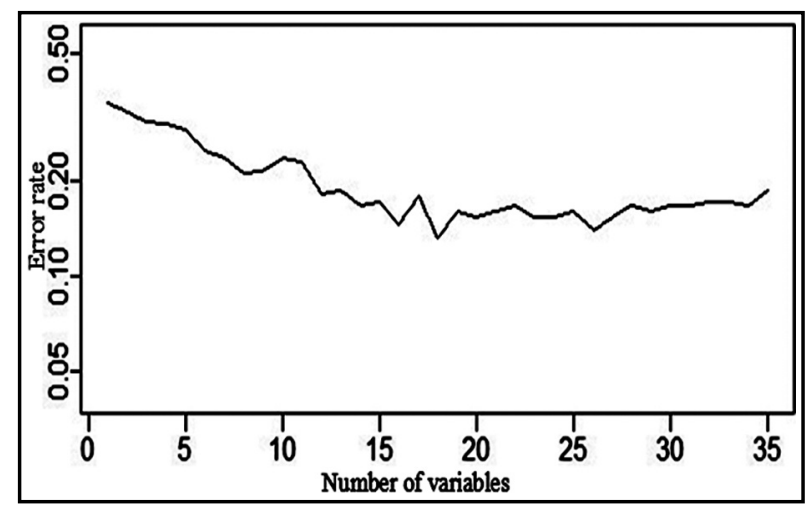

Fig.1: Classification error rate graph by the SWSFS process.

Abbreviations: SWSFS: sliding window sequential forward feature selection. 
Lai-Run Jin et al.

Table-II: Comparison of clinical signs between SLE-MS group and SLE-nMS group [n(\%)].

\begin{tabular}{|c|c|c|c|c|c|}
\hline Variables & & SLE-MS & SLE-nMS & $\chi^{2}$ & $P$ \\
\hline \multirow[t]{2}{*}{ Lupus nephritis } & Yes & 184(54.76) & $334(37.03)$ & 31.635 & $<0.001$ \\
\hline & No & $152(45.24)$ & $568(62.97)$ & & \\
\hline \multirow[t]{2}{*}{ Arthritis } & Yes & $122(36.3)$ & $417(46.2)$ & 9.802 & 0.002 \\
\hline & No & 214(63.7) & $485(53.8)$ & & \\
\hline \multirow[t]{2}{*}{ Rash } & Yes & 167(49.7) & $408(45.2)$ & 1.966 & 0.161 \\
\hline & No & $169(50.3)$ & $494(54.8)$ & & \\
\hline \multirow[t]{2}{*}{ Hair loss } & Yes & $33(9.8)$ & 123(13.6) & 3.235 & 0.072 \\
\hline & No & $303(90.2)$ & $779(86.4)$ & & \\
\hline \multirow[t]{2}{*}{ Proteinuria } & Yes & 237 (70.5) & $492(54.5)$ & 25.854 & $<0.001$ \\
\hline & No & $99(29.5)$ & $410(45.5)$ & & \\
\hline \multirow[t]{2}{*}{ Oral ulcer } & Yes & $47(14.0)$ & $87(9.6)$ & 4.783 & 0.029 \\
\hline & No & $289(86.0)$ & $815(90.4)$ & & \\
\hline \multirow[t]{2}{*}{ Fever } & Yes & 109(32.4) & $331(36.7)$ & 1.935 & 0.164 \\
\hline & No & $227(67.6)$ & $571(63.3)$ & & \\
\hline \multirow[t]{2}{*}{ Thrombocytopenia } & Yes & $122(36.3)$ & $282(31.3)$ & 2.835 & 0.092 \\
\hline & No & $214(63.7)$ & $620(68.7)$ & & \\
\hline \multirow[t]{2}{*}{ Leukopenia } & Yes & $239(71.1)$ & $628(69.6)$ & 0.265 & 0.607 \\
\hline & No & $97(28.9)$ & 274(30.4) & & \\
\hline \multirow[t]{2}{*}{ Headache } & Yes & $13(3.9)$ & $30(3.3)$ & 0.215 & 0.643 \\
\hline & No & $323(96.1)$ & 872(96.7) & & \\
\hline \multirow[t]{2}{*}{ Epilepsy } & Yes & $3(0.9)$ & $3(0.3)$ & - & 0.353 * \\
\hline & No & 333(99.1) & 899(99.7) & & \\
\hline \multirow[t]{2}{*}{ Brain syndrome } & Yes & $4(1.2)$ & $5(0.6)$ & - & 0.264 * \\
\hline & No & $332(98.8)$ & 897(99.4) & & \\
\hline \multirow[t]{2}{*}{ Mental illness } & Yes & 6(1.8) & 10(1.1) & - & 0.396 * \\
\hline & No & $330(98.2)$ & 892(98.9) & & \\
\hline \multirow[t]{2}{*}{ Optic neuritis } & Yes & $1(0.3)$ & $0(0)$ & - & 0.217 * \\
\hline & No & $335(99.7)$ & $902(100)$ & & \\
\hline \multirow[t]{2}{*}{ Cerebral neuritis } & Yes & $0(0)$ & $5(0.56)$ & - & 0.332 * \\
\hline & No & $336(100)$ & 897(99.4) & & \\
\hline \multirow[t]{2}{*}{ Cerebrovascular disease } & Yes & $1(0.3)$ & $0(0)$ & - & 0.271 * \\
\hline & No & $335(99.7)$ & $902(100)$ & & \\
\hline \multirow[t]{2}{*}{ Vasculitis } & Yes & $1(0.3)$ & $1(0.1)$ & - & 0.469 * \\
\hline & No & 335(99.7) & 901(99.9) & & \\
\hline \multirow[t]{2}{*}{ Tubular urine } & Yes & $37(11.0)$ & $44(4.9)$ & 15.063 & $<0.001$ \\
\hline & No & 299(89.0) & $858(95.1)$ & & \\
\hline \multirow[t]{2}{*}{ Hematuria } & Yes & $126(37.5)$ & $251(27.8)$ & 10.815 & 0.001 \\
\hline & No & $210(62.5)$ & $651(72.2)$ & & \\
\hline
\end{tabular}

Note: Significant results are highlighted in bold type. * indicates the P value obtained by Fisher's exact test.

We established a random forest model with or without MS as the dependent variable, and the statistically significant index after the univariate analysis as the independent variable. The results showed that the top 18 variables ranked by importance score were: BMI, SBP, DBP, Glu, UP, arthritis, occupation, UA, TG, education, HDL, WBC, IgG, C3, LDL, MCV, SLEDAI, GLB (TableIV). We ran the SWSFS process and plotted the error rate graph. The results showed that the error rate is the lowest when the number of independent variables is 18 (Fig.1).

We performed binary logistic regression analysis with or without MS as the dependent variable and the top 18 indicators of variable importance score as independent variables. The results showed that BMI, DBP, SBP, GLU, arthritis, UA, TG, and HDL were associated with MS in SLE (Table-V).

\section{DISCUSSIONS}

The clinical manifestations of almost all autoimmune diseases are likely to occur in SLE. Therefore, most scholars call it the prototype of autoimmune diseases, and its pathogenesis is still unclear. ${ }^{15}$ MS is related to the occurrence of CVD in SLE patients. Preventing the occurrence of MS in SLE patients is beneficial to the control of their condition. This study showed that the prevalence 
of MS in SLE patients is $27.14 \%$, which is lower than the results of scholars in South America and Europe, ${ }^{16,17}$ which may be related to the different samples from different regions and the diagnostic criteria used. The results of our previous survey showed that the MS prevalence rate of community physical examination population was $13.4 \%,{ }^{18}$ so the MS prevalence rate of SLE patients was significantly higher than that of community physical examination population. SLE is an important public health problem in the world. MS is an independent risk factor for CVD in SLE. Exploring the related factors of MS in SLE patients is of great significance for controlling the occurrence and development of SLE.

This study showed that MS in SLE patients is associated with farmers, married, and low levels of education. This may be because farmers have

Table-III: Comparison of Physical examination and laboratory

parameters between SLE-MS group and SLE-nMS group.

\begin{tabular}{|c|c|c|c|c|}
\hline Variables & SLE-MS & $S L E-n M S$ & $t / Z$ & $P$ \\
\hline Body mass index (BMI) & $23.59 \pm 3.83$ & $20.53 \pm 2.07$ & 13.896 & $<0.001$ \\
\hline SLE disease activity index (SLEDAI) & $10(7,13)$ & $9(5,13)$ & 3.389 & 0.001 \\
\hline Systolic blood pressure (SBP) & $138(123,150)$ & $120(110,130)$ & 12.888 & $<0.001$ \\
\hline Diastolic blood pressure (DBP) & $90(80,100)$ & $80(70,86)$ & 12.438 & $<0.001$ \\
\hline White blood cell count (WBC) & $5.32(3.73,8.30)$ & $4.80(3.70,7.10)$ & 2.207 & 0.027 \\
\hline Red blood cell count (RBC) & $3.87(3.71,4.28)$ & $3.97(3.64,4.25)$ & 0.714 & 0.475 \\
\hline Neutrophil percentage & $69.2(63.1,77.85)$ & $70.2(61.0,77.22)$ & 0.175 & 0.861 \\
\hline Hemoglobin amount & $113.0(100.32,124.50)$ & $114.0(103.02,125.00)$ & 1.043 & 0.297 \\
\hline Hematocrit & $0.35(0.32,0.39)$ & $0.354(0.324,0.390)$ & 0.689 & 0.491 \\
\hline Mean red blood cell volume (MCV) & $88.5(84.20,91.70)$ & $85.70(90.10,94.80)$ & 4.401 & $<0.001$ \\
\hline Red blood cell distribution width (RDW) & $14.25(13.50,16.40)$ & $14.10(13.10,15.40)$ & 2.455 & 0.014 \\
\hline Platelet count & $137.75(97.50,180.00)$ & $140.0(102.0,188.0)$ & 0.351 & 0.726 \\
\hline Platelet distribution width (PDW) & $16.40(15.60,17.30)$ & $15.30(16.57,17.10)$ & 0.469 & 0.639 \\
\hline Urine microalbumin & $0.18(0.15,0.54)$ & $0.15(0.00,0.26)$ & 3.667 & $<0.001$ \\
\hline Urine white blood cell count (UWBC) & $17.30(5.30,55.60)$ & $13.60(4.70,41.03)$ & 1.423 & 0.155 \\
\hline Urine red blood cell count (URBC) & $18.90(5.90,67.60)$ & $11.80(4.70,37.05)$ & 3.559 & $<0.001$ \\
\hline Urinary tube type count (UTT) & $0.64(0.14,1.41)$ & $0.51(0.13,1.30)$ & 2.103 & 0.035 \\
\hline Total protein $(\mathrm{TP})$ & $61.25(53.10,68.10)$ & $65.40(60.00,71.00)$ & 5.584 & $<0.001$ \\
\hline Triglyceride (TG) & $1.89(1.33,2.68)$ & $1.26(0.93,1.61)$ & 10.677 & $<0.001$ \\
\hline Albumin (ALB) & $33.50(26.70,38.00)$ & $35.60(30.50,39.70)$ & 4.242 & $<0.001$ \\
\hline High density lipoprotein (HDL) & $1.03(0.83,1.35)$ & $1.17(0.90,1.46)$ & 2.797 & 0.005 \\
\hline Globulin (GLB) & $26.60(22.20,32.40)$ & $29.10(25.10,34.00)$ & 4.096 & $<0.001$ \\
\hline Low density lipoprotein (LDL) & $2.27(1.71,3.17)$ & $2.15(1.63,2.80)$ & 2.627 & 0.009 \\
\hline White ball ratio (WBR) & $1.20(0.90,1.50)$ & $1.20(1.00,1.40)$ & 0.025 & 0.980 \\
\hline Aspartate aminotransferase (AST) & $19.00(12.00,32.25)$ & $17.00(11.00,29.00)$ & 2.163 & 0.031 \\
\hline C-reactive protein (CRP) & $5.91(2.20,15.90)$ & $4.72(2.08,18.80)$ & 0.537 & 0.591 \\
\hline Alanine aminotransferase (ALT) & $22.00(16.00,32.00)$ & $21.00(17.00,31.00)$ & 0.062 & 0.951 \\
\hline Complement 3 (C3) & $0.82(0.55,1.06)$ & $0.73(0.54,0.95)$ & 2.000 & 0.045 \\
\hline Urea (UA) & $6.96(4.68,11.74)$ & $4.78(3.68,6.57)$ & 7.883 & $<0.001$ \\
\hline Complement 4 (C4) & $0.14(0.06,0.24)$ & $0.14(0.07,0.22)$ & 0.248 & 0.804 \\
\hline Creatinine (CREA) & $72.60(53.65,136.88)$ & $59.60(48.55,74.98)$ & 6.029 & $<0.001$ \\
\hline Immunoglobulin A (IgA) & $2.43(1.71,3.31)$ & $2.63(2.01,3.54)$ & 2.258 & 0.024 \\
\hline Fasting blood glucose (GLU) & $5.85 \pm 2.50$ & $4.91 \pm 1.69$ & 5.857 & $<0.001$ \\
\hline Immunoglobulin G (IgG) & $12.60(9.42,17.60)$ & $15.30(11.57,19.40)$ & 4.585 & $<0.001$ \\
\hline Total cholesterol (TC) & $4.59(3.44,5.85)$ & $3.97(3.23,4.85)$ & 5.153 & $<0.001$ \\
\hline Immunoglobulin $\mathrm{M}(\mathrm{IgM})$ & $0.91(0.50,1.34)$ & $0.96(0.60,1.44)$ & 1.437 & 0.151 \\
\hline Immunoglobulin E (IgE) & $57.88(18.25,212.22)$ & $56.70(16.46,189.75)$ & 0.733 & 0.464 \\
\hline Erythrocyte sedimentation rate (ESR) & $40.40(19.85,72.00)$ & $37.05(17.00,67.40)$ & 1.598 & 0.110 \\
\hline $24 \mathrm{~h}$ urine protein (UP24) & $1.14(0.29,2.44)$ & $0.34(0.12,1.38)$ & 4.777 & $<0.001$ \\
\hline
\end{tabular}

Note: Significant results are highlighted in bold type. 
Table-IV: Variable importance score by random forest algorithm.

\begin{tabular}{lc}
\hline Variables & Importance score \\
\hline BMI & 10.28 \\
DBP & 9.39 \\
SBP & 7.39 \\
GLU & 4.31 \\
UP & 3.41 \\
Arthritis & 2.93 \\
Occupation & 2.22 \\
UA & 1.59 \\
TG & 1.59 \\
Education & 1.58 \\
HDL & 1.56 \\
WBC & 1.45 \\
IgG & 1.38 \\
C3 & 1.28 \\
LDL & 1.22 \\
MCV & 1.18 \\
SLEDAI & 1.17 \\
GLB & 1.06 \\
IgA & 1.05 \\
CREA & 1.03 \\
LN & 1.01 \\
UP24 & 0.94 \\
Mouthulcer & 0.93 \\
ALT & 0.93 \\
RDW & 0.89 \\
ALB & 0.80 \\
TP & 0.78 \\
Proteinuria & 0.72 \\
TC & 0.71 \\
URBC & 0.69 \\
Urine microalbumin & 0.68 \\
UTT & 0.58 \\
Cylindruria & 0.52 \\
Marriage & 0.48 \\
\hline Abbreviations: & 0.46 \\
\hline
\end{tabular}

Abbreviations: BMI: body mass index; DBP: diastolic blood pressure; SBP: systolic blood pressure; GLU: fasting blood glucose; UP: Urinary protein; UA: urea; TG: triglyceride; HDL: high density lipoprotein; WBC: white blood cell count; IgG: immunoglobulin G; C3: complement 3; LDL: low density lipoprotein; $\mathrm{MCV}$ : mean red blood cell volume; SLEDAI: systemic lupus erythematosus disease activity index; GLB: globulin; IgA: immunoglobulin A; CREA: creatinine; LN: lupus nephritis; UP24: 24h urine protein.

a relatively low level of education, low economic income, poor self-care awareness, and less access to health knowledge. Haiyan Shi et al. found that the middle-aged population generally lacks knowledge of MS and has not even heard of MS. ${ }^{19}$ Therefore, the prevention and treatment of MS in SLE patients should focus on farmers and low-education populations, and their health knowledge promotion and health services should be strengthened.

Lupus nephritis (LN) is a serious and common complication of SLE, which can significantly increase the incidence and mortality of SLE. ${ }^{20}$ This study found that the occurrence of MS in SLE patients is related to LN, which is consistent with the results of Zhang $\mathrm{M}$ et al. ${ }^{21} \mathrm{LN}$ patients have a variety of autoimmune lymphocytes and autoantibodies, which can activate endothelial cells and cause CVD. Therefore, LN itself is a risk factor for CVD in SLE patients. The risk factors of LN include family history, smoking history, age, etc. At the same time, the components of blood glucose, blood pressure, blood lipids and body weight in MS interact with each other in metabolism, which is also an important risk factor for LN. Moreover, the proportion of arthritis, proteinuria, mouth ulcer, tubular urine and hematuria in SLE-MS patients was significantly different from that in SLE-nMS group $(P<0.05)$, suggesting that symptomatic treatment of these signs in clinical practice is of great significance for the prevention and control of MS in SLE.

Red blood cell distribution width (RDW) is a parameter that directly describes red blood cell heterogeneity and is generally used to differentially diagnose thalassemia, giant cell anemia, and iron deficiency anemia. Recently, RDW has been found to be a strong independent predictor of allcause mortality in coronary atherosclerotic heart disease (CAD), acute coronary syndrome (ACS), hypertension, and diabetic nephropathy.22,23 Univariate analysis of this study showed that RDW was associated with SLE-MS, similar to our previous survey of metabolic syndrome in community physical examination populations, suggesting that RDW, a simple, inexpensive and readily available parameter, has good early warning and prognostic evaluation values in SLE-MS. ${ }^{18}$ The univariate analysis of this study showed that SLEDAI was associated with MS in SLE, indicating that MS may affect the severity of SLE disease.

Multivariate logistic regression analysis of this study showed that BMI, SBP, DBP, GLU, TG, and HDL were related to MS in SLE. These reflect the relationship between obesity, hypertension, hyperglycemia, dyslipidemia and MS in the general population also exists in SLE patients. ${ }^{24,25}$ Multivariate analysis showed that arthritis was a risk factor for MS in SLE. Lee et al. found 
Table-V: Binary logistic regression analysis of influencing factors regarding MS in SLE.

\begin{tabular}{lccccc}
\hline Independent variable & $\beta$ & $s \bar{x}$ & Wald $\chi^{2}$ & $P$ & OR (95\%CI) \\
\hline Body mass index (BMI) & 0.443 & 0.079 & 31.408 & $<0.001$ & $1.557(1.334,1.818)$ \\
Diastolic blood pressure (DBP) & 0.07 & 0.017 & 16.233 & $<0.001$ & $1.073(1.037,1.110)$ \\
Systolic blood pressure (SBP) & 0.031 & 0.012 & 7.014 & 0.008 & $1.031(1.008,1.055)$ \\
Fasting blood glucose (GLU) & 1.338 & 0.208 & 41.478 & $<0.001$ & $3.813(2.537,5.730)$ \\
Arthritis & 1.385 & 0.389 & 12.656 & $<0.001$ & $3.995(1.863,8.569)$ \\
Urea (UA) & 0.071 & 0.03 & 5.444 & 0.020 & $1.074(1.011,1.139)$ \\
Triglyceride (TG) & 0.86 & 0.18 & 22.904 & $<0.001$ & $2.364(1.662,3.362)$ \\
High density lipoprotein (HDL) & -1.812 & 0.504 & 12.929 & $<0.001$ & $0.163(0.061,0.439)$ \\
White blood cell count (WBC) & 0.09 & 0.052 & 3.709 & 0.079 & $1.095(0.989,1.211)$ \\
Constant & -29.406 & 3.385 & 75.455 & $<0.001$ & $<0.001$ \\
\hline
\end{tabular}

Note: Significant results are highlighted in bold type.

that the prevalence of MS in healthy controls was significantly lower than in patients with rheumatoid arthritis and the results support the views of this paper. ${ }^{26}$

Limitations of study: Several limitations exist in the current study. The lifestyle and dietary habits of the subjects in this study were not analyzed, so they need to be improved in future work. The patients with SLE in this study were recruited from only one hospital and the promotion of conclusions needs to consider regional factors. In addition, distorted results in epidemiological association studies can be resulted in by potential biases in case-control studies. Consequently, further studies are needed to clarify the exact influencing factors on MS in SLE.

\section{CONCLUSION}

The occurrence of MS in SLE patients is closely related to BMI, blood pressure, blood sugar, blood lipids, and arthritis.

Acknowledgement: Special thanks to Zhimin Shao for her help and assistance.

Funding: This study was funded by the National Science Foundation of Anhui Province, China (Grant Number: 1608085MH219 and 1808085QH251).

\section{Declaration of interest: None.}

\section{REFERENCES}

1. Salgado A, Herrera-Diaz C. Lupus Nephritis: An Overview of Recent Findings. Autoimmune Dis. 2014;2012(1):849684. doi: 10.1155/2012/849684

2. Sule S, Fontaine K. Abnormal body composition, cardiovascular endurance, and muscle strength in pediatric SLE. Pediatr Rheumatol Online J. 2016;14(1):50. doi: 10.1186/s12969-016-0110-8
3. Parker B, Urowitz MB, Gladman DD, Lunt M, Bae SC, Sanchez-Guerrero J, et al. Clinical associations of the metabolic syndrome in systemic lupus erythematosus: data from an international inception cohort. Ann Rheum Dis. 2013;72(8):1308. doi: 10.1136/annrheumdis-2012-202106

4. Ehrenthal DB, Haynes SG, Martin KE, Hitch JA, Addo SF, O'Neill E, et al. Evaluation of the Heart Truth, Professional Education Campaign on Provider Knowledge of Women and Heart Disease. Womens Health Issues. 2013;23(2):e87-e93. doi: 10.1016/j.whi.2013.01.001

5. Tektonidou MG, Kravvariti E, Konstantonis G, Tentolouris $\mathrm{N}$, Sfikakis PP, Protogerou A. Subclinical atherosclerosis in Systemic Lupus Erythematosus: Comparable risk with Diabetes Mellitus and Rheumatoid arthritis. Autoimmun Rev. 2017;16(3):308-312. doi: 10.1016/j.autrev.2017.01.009

6. Selzer F, Sutton-Tyrrell K, Fitzgerald SG, Pratt JE, Tracy $\mathrm{RP}$, Kuller LH, et al. Comparison of risk factors for vascular disease in the carotid artery and aorta in women with systemic lupus erythematosus. Arthritis Rheum. 2004;50(1):151. doi: 10.1002/art.11418

7. Tuttolomondo A, Di RD, Di SR, Pecoraro R, Arnao V, Butta C, et al. Arterial stiffness and ischemic stroke in subjects with and without metabolic syndrome. Atherosclerosis. 2012;225(1):216-219. doi: 10.1016/j. atherosclerosis.2012.08.027

8. Demir S, Artim-Esen B, Şahinkaya Y, Pehlivan O, AlpayKanitez N, Omma A, et al. Metabolic syndrome is not only a risk factor for cardiovascular diseases in systemic lupus erythematosus but is also associated with cumulative organ damage: a cross-sectional analysis of 311 patients. Lupus. 2016;25(2):177-184. doi: 10.1177/0961203315603140

9. Sule S, Fontaine K. Abnormal body composition, cardiovascular endurance, and muscle strength in pediatric SLE. Pediatr Rheumatol Online J. 2016;14(1):50. doi: 10.1186/ s12969-016-0110-8

10. Demir S, Artim-Esen B, Sahinkaya Y, Pehlivan O, AlpayKanitez N, Omma A, et al. Metabolic syndrome is not only a risk factor for cardiovascular diseases in systemic lupus erythematosus but is also associated with cumulative organ damage: A cross-sectional analysis of 311 patients. Lupus. 2016;25(2):177-184. doi: 10.1177/0961203315603140

11. Hochberg MC. Updating the American college of rheumatology revised criteria for the classification of systemic lupus erythematosus. Arthritis Rheum. 1997;40:1725. doi: 10.1002/art.1780400928

12. Gladman DD, Ibañez D, Urowitz MB. Systemic lupus erythematosus disease activity index 2000. J Rheumatol. 2002;29:288-291. 
13. Chinese diabetes society. Advice on metabolic syndrome by the Chinese diabetes society. Chin J Diabetes. 2004;12(3):156-161. doi: 10.1002/ijgo.12802

14. Jiang $\mathrm{R}$, Tang $\mathrm{W}, \mathrm{Wu} \mathrm{X}, \mathrm{Fu} \mathrm{W}$. A random forest approach to the detection of epistatic interactions in case-control studies. Bmc Bioinformatics. 2009;10(Suppl 1):1-12. doi: 10.1186/1471-2105-10-S1-S65

15. Yu M, Lu B, Liu Y, Me Y, Wang L, Li H. Interference with Tim-3 protein expression attenuates the invasion of clear cell renal cell carcinoma and aggravates anoikis. Mol Med Rep. 2017;15(3):1103. doi: 10.3892/mmr.2017.6136

16. Medeiros MM, Xavier de Oliveira ÍM, Ribeiro ÁT, RIBEIRO AT. Prevalence of metabolic syndrome in a cohort of systemic lupus erythematosus patients from Northeastern Brazil: association with disease activity, nephritis, smoking, and age. Rheumatol Int. 2016;36(1):117-124. doi: 10.1007/s00296-015-3316-z

17. Bellomio V, Spindler A, Lucero E, Berman A, Sueldo $\mathrm{R}$, Berman $\mathrm{H}$, et al. Metabolic syndrome in Argentinean patients with systemic lupus erythematosus. Lupus. 2009;18(11):1019-1025. doi: 10.1177/0961203309105876

18. Sun H, JIN L, Zuo X, Yuan H, Ding L, Shi W. Study on the prevalence of metabolic syndrome and its influencing factors in a community medical check-up crowd. Chin J Dis Control Prev. 2017;21(5):465-468. [Chinese]

19. Shi H, Wang J, Sai X. Cross-sectional investigation on the effects of metabolic syndrome related factors in middleaged population. Chin J Health Care Med. 2018;20(4):286288. [Chinese]

20. Simmons SC, Smith ML, Chang-Miller A, Keddis MT. Antinuclear Antibody-Negative Lupus Nephritis with Full House Nephropathy: A Case Report and Review of the Literature. Am J Nephrol. 2015;42(6):451-459. doi: $10.1159 / 000443747$

21. Zhang M, Qi C, Cao L, Qian J, Ni Z. Metabolic Syndrome is Correlated with Carotid Atherosclerosis in Patients with Lupus Nephritis. Am J Med Sci. 2014;348(6):486-491. doi: 10.1097/MAJ.0000000000000343

22. Jiang $\mathrm{M}, \mathrm{Zha} X, \mathrm{Wu} \mathrm{Z}, \mathrm{Zhu} X, \mathrm{Li} W, \mathrm{Wu} H$. Inverted U-shaped curve relationship between red blood cell distribution width and hypertension in a large health checkup population in China. J Am Soc Hypertens. 2018;12(5):327-334. doi: 10.1016/j.jash.2018.03.003
23. Hu Z, Sun $Y$, Wang $Q$, Han Z, Huang $Y$, Liu X, et al. Red blood cell distribution width is a potential prognostic index for liver disease. Clin Chem Lab Med. 2013;51(7):14031408. doi: $10.1515 /$ cclm-2012-0704

24. Bozkurt B, Aguilar D, Deswal A, Dunbar SB, Francis GS, Horwich T. et al. Contributory Risk and Management of Comorbidities of Hypertension, Obesity, Diabetes Mellitus, Hyperlipidemia, and Metabolic Syndrome in Chronic Heart Failure: A Scientific Statement From the American Heart Association. Circulation. 2016;134(23):e535-e578. doi: 10.1161/CIR.0000000000000450

25. Mahmood SN, Mukhtar KN, Deen S, Khan FN. Renal Biopsy: A much needed tool in patients with Systemic Lupus Erythematosis (SLE). 2016;32(1):70-74. doi: $10.12669 /$ pjms.321.3386

26. Lee SG, Kim JM, Lee SH, Kim KH, Kim JH, Yi JW, et al. Is the frequency of metabolic syndrome higher in South Korean women with rheumatoid arthritis than in healthy subjects? Korean J Intern Med. 2013;28(2):206-215. doi: 10.3904/kjim.2013.28.2.206

\section{Authors' Contributions:}

HY designed the study and conceived the survey, evaluated the results and revised the manuscript.

JZ and MJT collected epidemiological data.

LX and HP sorted the data.

QL and ZL were involved in the fieldwork.

LRJ conducted the analysis and wrote the first draft of the manuscript.

All authors approved the final version of the manuscript and contributed toward data analysis, drafting and revising the paper and agree to be accountable for all aspects of the work.
Authors:

1. Lai-Run Jin,

Office of Educational Administration,

2. Meng-Jun Tao,

School of Public Health,

3. Jun Zhou,

School of Public Health,

4. Liang $\mathrm{Xu}$

Department of Rheumatology,

5. Qiang Li,

Department of Medical Record,

6. Zhi Li,

Department of Rheumatology,

7. Hui Peng,

Administration Office of Hospital Admission and Discharge,

8. Hui Yuan,

School of Public Health,

1,4-7: The First Affiliated Hospital of Wannan Medical College (Yijishan Hospital of Wannan Medical College), Wuhu 241001, China.

2,3,8: Wannan Medical College, Wuhu 241001, China. 